\title{
QUALIDADE DE VIDA E FATORES DE RISCO À SAÚDE DE CUIDADORAS FORMAIS DE IDOSOS
}

\author{
Edison dos Reis ${ }^{1}$ \\ Victor Zuniga Dourado² \\ Ricardo Luís Fernandes Guerra ${ }^{3}$
}

resumo

O objetivo deste estudo foi avaliar e correlacionar a percepção da qualidade de vida e fatores de risco à saúde de cuidadores formais de idosos. A coleta de dados ocorreu por meio de formulário para caracterização sociodemográfica, avaliação antropométrica, da composição corporal e da percepção da qualidade de vida (SF-36). A amostra constitui-se de 34 cuidadoras concluintes do curso livre de capacitação em saúde, com idade média de 43,68 \pm 10,86 anos, sendo que $82 \%$ das cuidadoras possuíam 9 ou mais anos de escolari-

\footnotetext{
1 Enfermeiro. Mestre pelo Programa de Pós-Graduação Interdisciplinar em Ciências da Saúde da Universidade Federal de São Paulo (Unifesp). E-mail: edson.ufscar@gmail.com.

2 Fisioterapeuta. Doutor em Fisiopatologia em Clínica Médica pela Universidade Estadual Paulista (Unesp). Professor do Departamento de Ciências do Movimento Humano da Universidade Federal de São Paulo (Unifesp). E-mail: vzdourado@yahoo.com.br.

3 Profissional de Educação Física. Doutor em Ciências Fisiológicas pela Universidade Federal de São Carlos (UFSCar). Professor do Departamento de Ciências do Movimento Humano da Universidade Federal de São Paulo (Unifesp). E-mail: ricoguerra06@yahoo.com.br.
} 
dade. Os dados obtidos indicaram que 80\% da amostra encontrava-se com sobrepeso ou obesidade, 33\% com hipertensão arterial, 85\% com a circunferência da cintura (CC) elevada ou muito elevada, 62\% com circunferência do pescoço (CP) aumentada e 74\% com a razão cintura/quadril (RCQ) aumentada. Além disso, observou-se valores baixos nos domínios: Dor, Estado do Geral de Saúde e Vitalidade relativos à percepção da qualidade de vida. Ao correlacionar a variável CC com as demais variáveis, verificou-se: forte correlação com o índice de massa corporal (IMC), a CP e a porcentagem de gordura corporal; correlação moderada com as variáveis Massa Magra, Pressão Arterial e RCQ; e fraca correlação com a variável Média Geral do SF 36. Assim, verificamos que existe alta prevalência dos fatores de risco relacionados a doenças crônicas não transmissíveis (DCNT), doenças cardiovasculares (DCV) e síndrome metabólica, além de correlações entre as variáveis estudadas, evidenciando a necessidade de intervenções interdisciplinares e políticas de saúde voltadas para esse grupo específico de trabalho.

palavras-chave

Cuidadores. Composição Corporal. Qualidade de Vida. Risco Cardiovascular. Idoso.

O envelhecimento populacional é uma realidade no Brasil e no mundo, contudo, esse processo envolve alterações fisiológicas que tornam os idosos mais vulneráveis (GRATÃO et al., 2013). As possíveis consequências são as perdas e/ou redução no nível da autonomia e/ou independência (SEIMA; LENARDT, 2011; ROCHA; PACHECO, 2013).

As limitações dos idosos nas tarefas da vida diária passam a necessitar de auxílio para sua execução, além disso, a composição das famílias vem sofrendo redução em seus componentes, implicando na obtenção de uma ajuda qualificada (ROCHA; PACHECO, 2013), surge assim o papel do cuidador formal (AMENDOLA; OLIVEIRA; ALVARENGA, 2011). O cuidador formal, ao contrário do informal, é aquele que desempenha cuidado profissional, com remuneração, embora, ainda não seja uma profissão regulamentada (BATISTA; ALMEIDA; LANCMAN, 2014). Nesse cenário, identifica-se o desgaste físico e emocional ao qual o cuidador é 
exposto (SHIRAI; KOERNER; KENYON, 2009), gerando fatores estressantes e o desenvolvimento de doenças crônicas não transmissíveis (GRATÃO et al., 2013).

Estudos apontam para o aumento das doenças crônicas, em especial, a obesidade e complicações do sobrepeso associado a fatores de risco, como: inatividade física, dieta aterogênica, estresse (socioeconômico e psicossocial), hipertensão e diabetes (THEME FILHA et al., 2015; MALTA et al., 2016). Neste contexto, alguns preditores de doenças crônicas podem ser levados em consideração, como: o índice de massa corporal $\left(\mathrm{IMC}=\right.$ massa corporal $/$ estatura $\left.^{2}\right)$ (RIBEIRO et al., 2011), marcadores antropométricos (OLIVEIRA et al., 2009), pressão arterial (TIBANA; BALSAMO; PRESTES, 2011).

Apesar do número de cuidadores formais estar aumentando devido ao aumento da população idosa, que necessita ou necessitará desse profissional em algum momento da vida (LINO et al., 2016), a qualidade de vida e fatores de risco à saúde dos mesmos ainda é pouco estudada não sabendo se estes apresentam valores normais em relação a variáveis antropométricas, de composição corporal, força e pressão arterial, informações estas que podem ser fundamentais para a saúde destes profissionais e seus pacientes ou clientes. O presente estudo sustenta a hipótese que cuidadoras formais de idosos apresentam fatores de risco aumentados assim como baixas pontuações relativas à percepção da qualidade de vida e que exista correlações fortes e moderadas entre as variáveis estudadas.

\section{Objetivo}

Avaliar e correlacionar a percepção da qualidade de vida e fatores de risco à saúde de cuidadores formais de idosos, oriundas do curso livre de capacitação em saúde.

\section{Métodos}

Trata-se de um estudo de abordagem quantitativa, de corte transversal e exploratório. O estudo foi desenvolvido no Centro de Cursos Livres/Curso de Capacitação Cuidadores de Idosos (CETECPG) em colaboração com o Laboratório de Ciências do Esporte/UNIFESP. A coleta de dados ocorreu após aprovação do projeto de pesquisa pelo Comitê de Ética em Pesquisa da UNIFESP/BS ( $n^{\circ}$ 1434/15) e autorização das instituições envolvidas. Os cuidadores voluntários assinaram o Termo de Consentimento Livre e Esclarecido (TCLE), antes de serem submetidos aos procedimentos do estudo. Ressalta-se 
que foram respeitados os preceitos éticos em pesquisa com seres humanos contidos na Resolução 466/2012 do Conselho Nacional de Pesquisa (CONEP).

\subsection{Amostra}

Foram convidados a participar do estudo todos os cuidadores que concluíram o curso livre de cuidador de idosos. Inicialmente, fez-se o contato com todo o grupo, 78 cuidadores, destes aceitaram participar e foram avaliadas 34 cuidadoras. Os critérios de inclusão foram cuidadores que aceitaram participar do estudo com idade igual ou maior de 18 anos. Os critérios de não inclusão foram: não apresentar condições físicas e psíquicas para executar as avaliações propostas no estudo e ter idade inferior a 18 anos.

Ao utilizarmos o termo cuidadores ao longo desse estudo, estaremos nos referindo aos cuidadores formais de idosos. No momento em que houver referência ao grupo de cuidadores informais, utilizaremos dessa nomenclatura.

\subsection{Antropometria}

As medidas antropométricas foram realizadas segundo o protocolo descrito em Petroski (2003). Foi mensurada a estatura (estadiômetro de madeira Sanny com precisão de $0,5 \mathrm{~cm}$ ), a massa corporal (MC) (Balança digital da marca Cauduro com capacidade de $180 \mathrm{~kg}$ e precisão de 100g). A circunferência de cintura (CC), quadril (CQ) e pescoço (CP) foram obtidas por meio de fita métrica simples, de tecido não elástico. A classificação da CC seguiu os seguintes parâmetros: $<80 \mathrm{~cm}$ normal, $80-88 \mathrm{~cm}$ aumentado, $>88 \mathrm{~cm}$ muito aumentado, para mulheres (NIH, 1998). Com base nos valores da CC e CQ, identificou-se a razão cintura-quadril (RCQ) utilizando o valor de 0,85 para mulheres como "normalidade" (POULIOT et al., 1994). A CP utilizou-se o seguinte valor: $<34 \mathrm{~cm}$ normal, para mulheres (NIH, 1998).

Posteriormente foram realizados os cálculos do índice de massa corporal $\left(\mathrm{IMC}=\right.$ massa corporal $(\mathrm{Kg}) /$ altura $\left(\mathrm{m}^{2}\right)$. A classificação do IMC seguiu os parâmetros utilizados pela $\mathrm{WHO}$ (1995), sendo $<18,5 \mathrm{~kg} / \mathrm{m}^{2}$ desnutrido, $18,5-24,9 \mathrm{~kg} / \mathrm{m}^{2}$ eutrófico, $25,0-29,9 \mathrm{~kg} / \mathrm{m}^{2}$ sobrepeso e acima de $30,0 \mathrm{~kg} / \mathrm{m}^{2}$ obesidade. 


\subsection{Análise da composição corporal}

Foi realizada por Bioimpedância, (impedância bioelétrica tetra-polar modelo Tanita Ironman Interscan - BC-558) e foram avaliadas: a porcentagem de gordura corporal total (\% Gord.), massa magra total (MM), a porcentagem de água $\left(\% \mathrm{H}_{2} \mathrm{O}\right)$, a taxa metabólica total $(\mathrm{TMB})$, a gordura visceral $(\mathrm{GV})$ e a densidade mineral óssea (DMO) de acordo com os preceitos de Heyward (2004).

\subsection{Aferição da pressão arterial (PA)}

Foi realizada com esfigmomanômetro aneróide e estetoscópio, obtida com o cuidador sentado e em repouso por mais de 5 minutos. Foi considerado hipertensos cuidadores que estavam em uso de medicação hipertensiva ou apresentaram valores de PA iguais ou acima de $140 / 90 \mathrm{mmHg}$, segundo a V Diretrizes Brasileiras de Hipertensão Arterial (2006).

\subsection{Avaliação da percepção da qualidade de vida}

Foi avaliada utilizando-se o questionário SF-36 - Pesquisa em Saúde, um Questionário Genérico de Avaliação da percepção da Qualidade de Vida (Medical Outcomes Study SF-36), traduzido e validado para população brasileira (CICONELLI et al., 1999). Trata-se de um instrumento multidimensional composto por 36 itens avaliando em 8 dimensões assim distribuídas: 10 itens relacionados com a capacidade funcional (CF); 4 itens de aspecto físicos (AF); 2 itens sobre dor; 5 itens relacionados com o estado geral de saúde (EGS); 4 itens sobre vitalidade (VI); 2 itens com relação aos limitação por aspectos sociais (AS); 3 itens sobre limitação por aspectos emocionais (AE); 5 itens relacionados com a saúde mental (SM) e mais uma questão de avaliação comparativa entre as condições de saúde atual e a de um ano atrás. Para avaliar os resultados, é determinado um escore para cada uma das questões que, posteriormente, são transformadas em escala de 0 a 100, onde "zero" corresponde a um pior estado de saúde e "cem" a um melhor estado. Cada uma das dimensões é analisada em separado. 


\subsection{Análise estatística}

Os dados coletados foram armazenados no programa Excel Windows 2010 e processados no software Statistical Package for Social Science (SPSS) versão 23.0. Receberam tratamento estatístico, descritivo e inferencial, sendo a análise descritiva apresentada em valores percentuais, média, desvio padrão e mediana. A análise inferencial foi realizada pelo Coeficiente de Correlação Linear de Pearson (r), para verificar associações entre as médias dos escores e as variáveis quantitativas. A classificação segundo a ordem de magnitude: 0,10 até 0,30 (fraca); 0,40 até 0,60 (moderada); 0,7 até 1 (forte) (DANCEY; REIDY, 2006). O nível de significância adotado para o estudo foi de $5 \%(p \leq 0,05)$.

\section{Resultados}

A amostra constitui-se de 34 cuidadores com idade média de 43,68 $\pm 10,86$, sendo que houve total predominância do gênero feminino 100\%, sendo assim, será utilizado o termo "cuidadora", no gênero feminino referindo-se às participantes do estudo. No item situação conjugal, $47 \%$ eram casadas, seguidas de solteiras $29 \%$. Em relação aos anos de escolaridade, $82 \%$ possuem 9 ou mais anos de estudo, na variável etnia, $50 \%$ se autodeclararam brancas. No item doenças crônicas, quando perguntado as cuidadoras se tinham pelo menos uma, $56 \%$ responderam que sim (Tabela 1 ).

Tabela 1 - Variáveis sociodemográficas ( $n$ = 34). Santos, SP, 2018.

\begin{tabular}{lll}
\hline Variáveis & N & $\%$ \\
\hline Idade & & \\
18 a 39 & 10 & $29 \%$ \\
40 a 59 & 22 & $65 \%$ \\
$\quad 60$ ou mais & 02 & $06 \%$ \\
Gênero & & \\
$\quad$ Masculino & 00 & $0 \%$ \\
$\quad$ Feminino & 34 & $100 \%$ \\
Situação Conjugal & & \\
$\quad$ Solteira & 10 & $29 \%$ \\
$\quad$ Casada/União Estável & 16 & $47 \%$ \\
Divorciada/Separada & 06 & $18 \%$ \\
Viúva & 02 & $06 \%$ \\
\hline
\end{tabular}




\begin{tabular}{lll}
\hline Variáveis & N & $\%$ \\
\hline Anos de Estudo & & \\
$\quad$ Analfabeta & 00 & $0 \%$ \\
De um a oito & 06 & $18 \%$ \\
De nove a onze & 23 & $68 \%$ \\
$\quad$ Doze ou mais & 05 & $14 \%$ \\
Etnia & & \\
Amarelo & 00 & $0 \%$ \\
Branco & 17 & $50 \%$ \\
Negro & 04 & $12 \%$ \\
Pardo & 13 & $38 \%$ \\
Doenças Crônicas & & \\
Sim & 18 & $53 \%$ \\
Não & 16 & $47 \%$ \\
\hline
\end{tabular}

Fonte: Dados da pesquisa, 2018

Na avaliação da percepção da QV apresentada na Tabela 2, as cuidadoras apresentaram maior pontuação no domínio Limitações por Aspectos Emocionais (LAE) com média de $83,23 \pm 24,96$ e mediana de 100,00 . O menor valor identificado foi no domínio DOR com média de 48,21 $\pm 24,88$ e mediada de 41,50, seguido dos domínios Estado Geral de Saúde e Vitalidade (Tabela 2).

Tabela 2 - Valores médios, desvio padrão e mediana dos escores do questionário de percepção de qualidade de vida - SF 36 ( $n=34)$. Santos, SP, 2018.

\begin{tabular}{lcc}
\hline Domínios do SF - 36 & Média (dp) & Mediana \\
\hline Capacidade Funcional (CF) & $82,79 \pm 16,77$ & 85,00 \\
Aspectos Físicos (LAF) & $74,85 \pm 25,88$ & 75,00 \\
Dor & $48,21 \pm 24,88$ & 41,50 \\
Estado Geral Saúde (EGS) & $60,79 \pm 13,95$ & 57,00 \\
Vitalidade N) & $63,38 \pm 15,28$ & 60,00 \\
Aspectos Sociais (AS) & $75,00 \pm 20,38$ & 75,00 \\
Limitações Aspectos Emocionais (LAE) & $83,23 \pm 24,96$ & 100,00 \\
Saúde Mental (SM) & $69,15 \pm 11,73$ & 72,00 \\
Média Geral & $69,53 \pm 10,71$ & 69,39 \\
\hline
\end{tabular}

Fonte: Dados da pesquisa, 2018.

$\mathrm{dp}=$ desvio-padrão; SF = Short Form.

Ao analisar a CC, observou-se que $85 \%$ das cuidadoras estavam acima do valor recomendado, sendo a média do grupo de $92,59 \mathrm{~cm} \pm 10,03$. Em relação 
à RCQ verificou-se que 74\% estavam com valores acima do normal, o mesmo para a variável CP com $62 \%$. Na avaliação do IMC, $47 \%$ estavam com sobrepeso e $32 \%$ com obesidade (Tabela 3 ).

Tabela 3 - Variáveis antropométricas e pressão arterial (n total = 34). Santos, SP, 2018.

\begin{tabular}{|c|c|c|}
\hline Variáveis & $\mathrm{N}$ & $\%$ \\
\hline Circunferência da Cintura (média \pm dp) & $92,59 \pm 10,03$ & \\
\hline $\begin{array}{l}\text { Classificação da CC } \\
\text { Normal } \\
\text { Elevado } \\
\text { Muito elevado }\end{array}$ & $\begin{array}{l}05 \\
06 \\
23\end{array}$ & $\begin{array}{l}15 \% \\
18 \% \\
67 \%\end{array}$ \\
\hline Razão Cintura/Quadril (média \pm dp) & $0,88 \pm 0,06$ & \\
\hline $\begin{array}{l}\text { Classificação da RCQ } \\
\text { Normal } \\
\text { Aumentada }\end{array}$ & $\begin{array}{l}09 \\
25\end{array}$ & $\begin{array}{l}26 \% \\
74 \%\end{array}$ \\
\hline Circunferência do Pescoço (média \pm dp) & $35,87 \pm 3,48$ & \\
\hline $\begin{array}{l}\text { Classificação da CP } \\
\text { Normal } \\
\text { Aumentada }\end{array}$ & $\begin{array}{l}13 \\
21\end{array}$ & $\begin{array}{l}38 \% \\
62 \%\end{array}$ \\
\hline $\begin{array}{l}\text { Pressão Arterial (média } \pm d p \text { ) PAS } \\
\text { PAD }\end{array}$ & $\begin{array}{c}125,88 \pm 10,03 \\
77,06 \pm 8,92\end{array}$ & \\
\hline $\begin{array}{l}\text { Classificação da PA } \\
\text { Normotenso } \\
\text { Hipertenso }\end{array}$ & $\begin{array}{l}23 \\
11\end{array}$ & $\begin{array}{l}67 \% \\
33 \%\end{array}$ \\
\hline IMC $\left(\mathrm{kg} / \mathrm{m}^{2}\right)$ (média $\left.\pm \mathrm{dp}\right)$ & $28,61 \pm 4,91$ & \\
\hline $\begin{array}{c}\text { Classificação do IMC } \\
\text { 18,5 - 24,9 Eutrófico } \\
\text { 25,0 - 29,9 Sobrepeso } \\
\text { > que 30,0 Obesidade I }\end{array}$ & $\begin{array}{l}07 \\
16 \\
11\end{array}$ & $\begin{array}{l}21 \% \\
47 \% \\
32 \%\end{array}$ \\
\hline
\end{tabular}

Fonte: Dados da pesquisa, 2018.

$\mathrm{dp}$ = desvio-padrão; IMC = Índice de Massa Corporal; Kg = Kilograma; PAS = Pressão Arterial Sistólica; PAD = Pressão Arterial Diastólica; m2 = metros ao quadrado.

Em relação aos valores da composição corporal, identificou-se média de $47,68 \pm 4,40 \%$ para a porcentagem de água corporal $(\% \mathrm{H} 2 \mathrm{O}), 35,37 \pm 6,49 \%$ para a porcentagem de gordura corporal total (\% G Total), $44,00 \pm 4,65 \mathrm{~kg}$ para massa magra total (MM) e 1415,09 $\pm 156,70 \mathrm{kcal}$ para a taxa metabólica basal (TMB). Já em relação a correlação entre a variável dependente $C C$ e as variáveis independentes (PA, MM, \% G total, CP e SF-36) observou-se que houve correlação forte e positiva com \% G total, $(\mathrm{r}=0,834 ; p<0,000), \mathrm{CP},(\mathrm{r}=0,810$; 
$p<0,000)$, e IMC, $(\mathrm{r}=0,730 ; p<0,000)$. As correlações moderadas ocorreram com as variáveis MM ( $\mathrm{r}=0,632 ; p<0,000)$, PA sistólica, $(\mathrm{r}=0,562 ; p<0,001) \mathrm{e}$ RCQ $(\mathrm{r}=0,676 ; p<0,000)$. A correlação com a variável média geral do SF-36, $(\mathrm{r}=-0,175 ; p<0,529)$ foi fraca.

\section{Discussão}

O objetivo do presente estudo foi avaliar e correlacionar a percepção da qualidade de vida $(\mathrm{QV})$ e fatores de risco à saúde dos cuidadores de idosos, visto que há uma demanda crescente por este profissional (BATISTA; ALMEIDA; LANCMAN, 2014) e também por conta do aumento da população idosa (ALMEIDA et al., 2015). Os resultados encontrados confirmam a hipótese sustentada de que as cuidadoras formais de idosos apresentam valores fora dos padrões indicados pela Organização Mundial de Saúde (OMS), para as variáveis antropométricas, de composição corporal e pressão arterial, logo, esse quadro favorece ao aumento de riscos à saúde (TORIMOTO-SASAI et al., 2015).

Estudos nacionais alertam para o crescimento das Doenças Crônicas Não Transmissíveis (DCNT) (BRASIL, 2011) e dos diversos fatores de risco que contribuem para o seu desenvolvimento, além disso, há preocupação em especial com o grupo das mulheres por apresentarem um quadro mais suscetível (MENDES et al., 2012; THEME FILHA et al., 2015).

É verdade que no último século as mulheres alcançaram diversas conquistas, dentre elas, o mercado de trabalho, em contrapartida ficaram mais expostas a desenvolverem doenças, em especial, DCNT. Ademais, a maioria das mulheres exercem tarefas cumulativas, que vão desde cuidados com os familiares e com a casa, educação dos filhos à atividade profissional (ALMEIDA et al., 2015).

Ao avaliar o perfil sociodemográfico das cuidadoras do presente estudo, percebe-se que os dados obtidos são condizentes com os achados na literatura, predominância do gênero feminino, com idade entre 40 a 50 anos (MOREIRA et al., 2018), maioria casadas/união estável, nível de escolaridade com mais de 9 anos de estudos (fundamental completo), e quando perguntado sobre ter alguma doença crônica, 53\% responderam que sim (SEIMA; LENARDT; CALDAS, 2014; SANTOS et al., 2014). Nesse sentido, ao analisar os valores da variável percepção da qualidade de vida $(\mathrm{QV})$, os domínios que despertam atenção foram, Limitações por Aspectos Emocionais (LAE) o qual apresentou o maior escore $(83,23 \pm 24,96$ e mediana 100,00$)$, o que nos leva a inferir que possíveis problemas emocionais não interferiram no exercício de suas atividades diárias. Por outro lado, os domínios com os menores escores (DOR, ESG e V), 
apontam consonância com a literatura, visto que, cuidadoras assumem com mais frequência tarefas desgastantes, como por exemplo, higiene do paciente, além da locomoção quando necessário (PINTO et al., 2009; NICKEL et al., 2010). Ao correlacionar a variável independente SF-36 com a variável dependente CC o resultado foi negativo e fraco, $(\mathrm{r}=-0175 ; p<0,529)$, sugerindo que, quanto melhor a percepção da qualidade de vida $(\mathrm{QV})$ menor é o valor da $C C$, contudo, o resultado obtido na correlação não permite afirmar fortemente tal suposição. Por outro lado, ao analisar outras correlações da variável dependente CC com as variáveis independentes, \% G total $(\mathrm{r}=0,834 ; p<0,000), \mathrm{CP}(\mathrm{r}=0,810$; $p<0,000)$ e IMC ( $\mathrm{r}=0,730 ; p<0,000)$ observou-se correlações positivas e fortes. Logo, perante esse cenário, pode-se constatar indicação de risco aumentado à saúde das cuidadoras, principalmente risco cardiovascular e metabólico, ainda que a média de idade não seja alta $(43,68 \pm 10,86$ anos). Aliado a este dado, deve-se considerar que mais da metade das avaliadas relataram possuir pelo menos uma doença crônica, além de valores antropométricos, de composição corporal e PA apresentarem-se acima do recomendado (POULIOT et al., 1994; WHO, 1995; BEN-NOUN; SOHAR; LAOR, 2001).

Ao refletir sobre a condição de saúde do grupo de cuidadoras e, em conjunto com as informações apresentadas pelo Ministério da Saúde, o qual afirma que quase metade da população brasileira está acima do peso, observa-se então cenário ainda mais preocupante uma vez que os resultados aqui identificados extrapolam os apresentados pela literatura, pois $47 \%$ das cuidadoras foram classificadas como tendo sobrepeso, e 32\% como sendo obesas. Isso significa que 79\% das voluntárias estão acima dos valores de normalidade (WHO, 1995), valores esses também superiores aos encontrados em estudos realizados com população adulta na cidade de Pelotas/RS (OLINTO et al., 2006) e Londrina/PR (PORTO et al., 2016), no qual 53\% e 64\% dos voluntários apresentavam sobrepeso/obesidade respectivamente, com $64 \%$ de sobrepeso/obesidade.

Autores apontam para associação entre sobrepeso/obesidade e deposição de gordura abdominal, visto que este é um fator de risco a saúde e indicador para o desenvolvimento de DCNT (OLIVEIRA et al., 2009). Além disso, essa condição torna o indivíduo mais suscetível a desenvolver disfunções metabólicas. No estudo de Mota et al. (2011), foi identificado que a variável CC alterada ou muito alterada, apresentou forte influência sobre anormalidades metabólicas relativas as concentrações de glicose, triacilglicerol, colesterol total, HDL e LDL.

Segundo Porto et al. (2016), a medida antropométrica da CC é um indicador de risco metabólico amplamente disseminado por diferentes entidades 
internacionais e nacionais. Portanto, quando está variável apresenta-se alterada, pode propiciar o surgimento de problemas cardiovasculares.

Outro resultado que chama atenção no presente estudo é que $62 \%$ das cuidadoras apresentaram CP aumentada, e quando correlacionada com IMC e CC obteve-se correlação forte e positiva. Segundo Preis et al. (2010), a CP aumentada pode ser indicativo de acúmulo de moléculas de gordura na parede das artérias carótidas, o que favorece a presença de doenças cardiovasculares (DCV). A mesma correlação foi realizada por Stabe et al. (2013), que identificaram haver associação entre $\mathrm{CP}$ aumentada com o IMC e CC, sendo um forte fator de risco para síndrome metabólica.

Além disso, a variável RCQ também contribui como fator de risco, pois segundo Frizon e Boscaini (2013) RCQ alta e/ou muito alta está associada às alterações metabólicas. No estudo de Souza et al. (2013), 55\% das mulheres com idade acima de 20 anos apresentaram RCQ elevada. Neste estudo identificamos que $74 \%$ das cuidadoras estão com RCQ aumentada, sendo esse um fator de risco para doenças cardiovasculares e metabólicas.

Ao observar os resultados apresentados, somados com as atividades desenvolvidas pelo cuidador (BORN, 2008), pode-se inferir que as cuidadoras com formação estejam mais sensíveis a compreender os impactos em relação aos fatores de risco: físicos, emocionais e sociais, sendo que Memoria, Carvalho e Rocha (2013) identificaram a falta de conhecimento do cuidador como um dos desafios a serem enfrentados neste contexto. Resultado esse que vai ao encontro do estudo de revisão realizado por Reis, Novelli e Guerra (2018) que concluíram que as intervenções não farmacológicas trouxeram benefícios à saúde das cuidadoras, ou seja, a capacitação do cuidador é um importante instrumento de enfrentamento para os diversos fatores de risco.

Este estudo apresenta limitações que incluem o modelo transversal, visto que um estudo longitudinal poderia avaliar de forma eficaz o comportamento das variáveis estudadas e, o tamanho da amostra é pequeno e foi composta por conveniência. Portanto, deve-se ter cautela ao extrapolar comparações generalizadas à população brasileira.

No entanto, o presente trabalho permite enxergarmos uma condição complexa para este grupo em específico. Como se propor a cuidar de alguém se a própria saúde requer cuidados? Assim como o número de idosos cresce, cresce também a necessidade de pessoas capacitadas para o cuidar, contudo, deve-se alertar para os fatores de risco a que essa população está exposta, pois o cuidar da saúde deve ser objetivo da díade cuidador/idoso. Nessa perspectiva, recente estudo (PLOEG et al., 2019) apontou para o fato de que os prestadores de cuidados em saúde devem considerar tanto o cuidador como o receptor 
do cuidado como clientes, facilitando a sua ligação com os serviços de apoio à saúde e à comunidade, auxiliando assim o entendimento da crescente complexidade das necessidades de cuidados, sendo que a adoção de tal abordagem requer coordenação social, econômica e política.

\section{Conclusão}

Conclui-se que houve identificação de alta prevalência dos fatores de risco analisados, demonstrados pelos indicativos de risco para IMC, CC, RC/Q, PA além de baixos scores para alguns domínios da qualidade de vida. Além disso, ao correlacionar a variável CC com as demais variáveis verificou-se forte correlação com IMC, CP e \% G total, correlação moderada com as variáveis MM, PA e RCQ. No entanto, verificou-se baixa correlação da CC com a variável de média geral do SF-36.

Apesar de serem escassos estudos que avaliaram fatores de risco à saúde de cuidadoras de idosos, o presente estudo permitiu inferir que as variáveis analisadas apresentaram alterações indicando comprometimentos a saúde da maioria das cuidadoras aqui avaliadas, cenário esse de grande relevância para o apontamento de novos estudos a partir de outros indicadores como, por exemplo, bioquímicos, nível de atividade física e hábitos alimentares, pois trata-se de fatores que evidenciam riscos para DCV e a saúde desta população, a qual se propõe a cuidar.

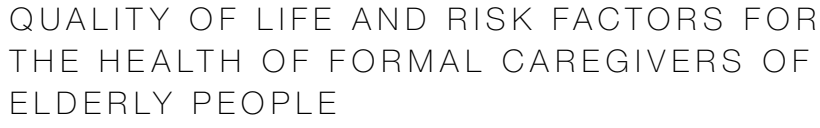

\section{abstract}

The aim of this study was to evaluate and correlate the perception of quality of life and risk factors to the health of elderly caregivers. Data were collected through a form for sociodemographic characterization, anthropometric, body composition and quality of life perception (SF-36) evaluation. The sample consisted of 34 caregivers who had completed the free training course on health, with a mean age of $43.68 \pm 10.86$ years old and $82 \%$ of caregivers have 9 or more years of schooling. The data indicated that $80 \%$ of the sample was overweight or obese, 33\% had hypertension, 85\% had the waist circumference (WC) high or very high, 62\% had an increased neck circumference (NC) and 74\% had the waist/hip (R W/H) increased. In 
addition, low values in Pain, General Health Status and vitality related to the perception of quality of life. When correlating the WC variable with the other variables, there was: a strong correlation with body mass index (BMI), NC and percentage of body fat; moderate correlation with the variables lean body mass, blood pressure and $\mathrm{R} W / H$, and; a weak correlation with the variable mean of QoL. Thus, this study indicates that there is high prevalence of risk factors related to chronic non-communicable diseases (CNCD), cardiovascular diseases (CVD) and metabolic syndrome, as well as correlations between the studied variables, evidencing the need for interdisciplinary interventions and health policies directed to this specific group of work.

keywords

Caregivers. Body Composition. Quality of Life. Cardiovascular Risk. Elderly.

referências

ALMEIDA, Alessandra Vieira et al. A feminização da velhice: em foco as características socioeconômicas, pessoais e familiares das idosas e o risco social. Textos \& Contextos, Porto Alegre, v. 14, n. 1, p. 115-131, 2015.

AMENDOLA, Fernanda; OLIVEIRA, Maria Amélia de Campos; ALVARENGA, Márcia Regina Martins. Influence of social support on the quality of life of family caregivers while caring for people with dependence. Revista da Escola de Enfermagem da USP, São Paulo, v. 45, n. 4, p. 884-889, 2011.

BATISTA, Marina Picazzio Perez; ALMEIDA, Maria Helena Morgani; LANCMAN, Selma. Cuidadores formais de idosos: contextualização histórica no cenário brasileiro. Revista Brasileira de Geriatria e Gerontologia, Rio de Janeiro, v. 17, n. 4, p. 879-885, 2014.

BEN-NOUN, Liubov; SOHAR, Ezra; LAOR, Arie. Neck circumference as a simple screening measure for identifying overweight and obese patients. Obesity Research, Baton Rouge, v. 9, n. 8, p. 470-477, 2001.

BORN, Tomiko (org.). Cuidar Melhor e Evitar a Violência: Manual do Cuidador da Pessoa Idosa. Brasília, DF: Secretaria Especial dos Direitos Humanos, Subsecretaria de Promoção e Defesa dos Direitos Humanos, 2008.

BRASIL. Ministério da Saúde. Secretaria de Vigilância em Saúde. Departamento de Análise de Situação de Saúde. Plano de ações estratégicas para o enfrentamento das doenças crônicas não transmissiveis (DCNT) no Brasil: 2011-2022. Brasília, DF: Ministério da Saúde, 2011.

CICONELLI, Rozana Mesquita et al. Tradução para a língua portuguesa e validação do questionário genérico de avaliação de qualidade de vida SF-36 (Brasil SF-36). Revista Brasileira de Reumatologia, Campinas, v. 39, n. 3, p. 143-150, 1999.

DANCEY, Christine P.; REIDY, John. Estatística sem matemática para psicologia: usando SPSS para Windows. 3. ed. Porto Alegre: Artmed, 2006.

FRIZON, Vanessa; BOSCAINI, Camile. Circunferência do pescoço, fatores de risco para doenças cardiovasculares e consumo alimentar. Revista Brasileira de Cardiologia, Rio de Janeiro, v. 26, n. 6, p. 426-434, 2013. 
GRATÃO, Aline Cristina Martins et al. Dependência funcional de idosos e a sobrecarga do cuidador. Revista da Escola de Enfermagem da USP, São Paulo, v. 47, n. 1, p. $1337-1344,2013$

HEYWARD, Vivian H. Avaliação da composição corporal.In: Avaliação Física e Prescrição de Exercício: Técnicas Avançadas. (Traduzido por Márcia Dornelles). 4. ed. Porto Alegre: Artmed, p. 143-170, 2004

LINO, Valéria Teresa Saraiva et al. Prevalência de sobrecarga e respectivos fatores associados em cuidadores de idosos dependentes, em uma região pobre do Rio de Janeiro, Brasil. Cadernos de Saúde Pública, Rio de Janeiro, v. 36, n. 6, p. 1-14, 2016.

MALTA, Deborah Carvalho et al. Avanços do Plano de Ações Estratégicas para o Enfrentamento das Doenças Crônicas Não Transmissiveis no Brasil, 2011-2015. Epidemiologia e Serviços de Saúde, Brasília, DF, v. 25, n. 2, p. 373-390, 2016.

MEMORIA, Larissa Vanessa Ferreira; CARVALHO, Maria Júlia Nascimento; ROCHA, Francisca Cecília Viana. A percepção do cuidador de idosos sobre o cuidado. Revista Interdisciplinar, Teresina, v. 6, n. 3, p. 15-25, 2013.

MENDES, Wanderson André Alves et al. Relação de variáveis antropométricas com os perfis pressórico e lipídico em adultos portadores de doenças crônicas não transmissíveis. Revista Brasileira de Cardiologia, Rio de Janeiro, v. 25, n. 3, p. 200-209, 2012.

MOREIRA, Andréa Carvalho Araújo et al. Efetividade da intervenção educativa no conhecimento-atitude-prática de cuidadores de idosos. Revista Brasileira de Enfermagem, Brasília, DF, v. 71, n. 3, p. 1118-1126, 2018

MOTA, João Felipe et al. Indicadores antropométricos como marcadores de risco para anormalidades metabólicas. Ciência \& Saúde Coletiva, Rio de Janeiro, v. 16, n. 9, p. 3901-3908, 2011

NATIONAL INSTITUTES OF HEALTH (NIH). Clinical guidelines on the identification, evaluation, and treatment of overweight and obesity in adults: the evidence report. Bethesda: NIH, 1998. (NIH Publication n. 98-4083).

NICKEL, Renato et al. Correlação entre a qualidade de vida de cuidadores familiares e os níveis de independência funcional dos cuidados. Cogitare Enfermagem, Curitiba, v. 15, n. 2 , p. 225-230, 2010

OLINTO, Maria Teresa Anselmo et al. Níveis de intervenção para a obesidade abdominal: prevalência e fatores associados. Cadernos de Saúde Pública, Rio de Janeiro, v. 22, n. 6, p. 1207-1215, 2006.

OLIVEIRA, Mirelle Arruda Michelotto et al. Relação de indicadores antropométricos com fatores de risco para doença cardiovascular. Arquivos Brasileiros de Cardiologia, São Paulo, v. 94, n. 4, p. 1-8, 2009.

PINTO, Meire Fernanda et al. Qualidade de vida de cuidadores de idosos com doença de Alzheimer. Acta Paulista Enfermagem, São Paulo, v. 22, n. 5, p. 652-657, 2009

PLOEG, Jenny et al. Caregivers of older adults with dementia and multiple chronic conditions: exploring their experiences with significant changes. Dementia, London, Mar. 2019. Disponível em: https://journals.sagepub.com/doi/full/10.1177/1471301219834423. Acesso em: 9 abr. 2019.

PORTO, Denilson Braga et al. Autopercepção de saúde em trabalhadores de um Hospital Universitário e sua associação com indicadores de adiposidade, pressão arterial e prática de atividade física. Ciência \& Saúde Coletiva, Rio de Janeiro, v. 21, n. 4, p. 1113-1122, 2016.

POULIOT, Marie-Christine et al. Waist circumference and abdominal sagittal diameter: best simple anthropometric indexes of abdominal visceral adipose tissue accumulation and related cardiovascular risk in men and women. American Journal of Cardiology, New York, v. 73, n. 7. p. 460-468, 1994. 
PREIS, Sara Rosner et al. Neck circumference as a novel measure of cardiometabolic risk: the Framingham Heart Study. Journal of Clinical Endocrinology \& Metabolism, Philadelphia, v. 95, n. 8, p. 3701-3710, 2010.

REIS, Edison dos; NOVELLI, Márcia Maria Pires Camargo; GUERRA, Ricardo Luís Fernandes. Intervenções realizadas com grupos de cuidadores de idosos com síndrome demencial: revisão sistemática. Cadernos Brasileiros de Terapia Ocupacional, São Carlos, v. 26, n. 3, p. 646-657, 2018.

RIBEIRO, Sandra Maria Lima et al. Análise vetorial de bioimpedância e estado nutricional de idosas de acordo com o índice de massa corporal. Revista Brasileira de Cineantropometria \& Desempenho Humano, Florianópolis, v. 13, n. 6, p. 415-421, 2011.

ROCHA, Bruno Miguel Parrinha; PACHECO, José Euzébio Palma. Idoso em situação de dependência: estresse e coping do cuidador informal. Acta Paulista Enfermagem, São Paulo, v. 26, n. 1, p. 50-56, 2013.

SANTOS, Raquel Luiza et al. Caregivers' quality of life in mild and moderate dementia. Arquivos de Neuro-psiquiatria, São Paulo, v. 72, n. 12, p. 931-937, 2014.

SEIMA, Marcia Daniele; LENARDT, Maria Helena. A sobrecarga do cuidador familiar de idoso com Alzheimer. Textos \& Contextos, Porto Alegre, v. 10, n. 2, p. 388-398, 2011

SEIMA, Marcia Daniele; LENARDT, Maria Helena; CALDAS, Célia Pereira. Relação no cuidado entre o cuidador familiar e o idoso com Alzheimer. Revista Brasileira de Enfermagem, São Paulo, v. 67, n. 2, p. 233-240, 2014.

SHIRAI, Yumi; KOERNER, Susan Silverberg; KENYON, Denyelle Baete. Reaping caregiver feelings of gain: the roles of socio-emotional support and mastery. Aging \& Mental Health Abingdon, v. 13, n. 1, p. 106-117, 2009

SOUZA, Aline Ozana et al. Associação dos indicadores antropométricos e sócio- demográficos ao risco de doença cardiovascular. Revista Paraense de Medicina, Belém, v. 27, n. 3, p. 45-54, 2013.

STABE, Christiane et al. Neck circumference as a simple tool for identifying the metabolic syndrome and insulin resistance: results from the Brazilian Metabolic Syndrome study (BRAMS). Clinical Endocrinology, Oxford. v. 78, n. 6, p. 874-881, 2013.

THEME FILHA, Mariza Miranda et al. Prevalência de doenças crônicas não transmissíveis e associação com autoavaliação de saúde: Pesquisa Nacional de Saúde, 2013. Revista Brasileira de Epidemiologia, São Paulo, v. 18, n. 2, p. 83-96, 2015.

TIBANA, Ramires Alsamir; BALSAMO, Sandor; PRESTES, Jonato. Associação entre força muscular relativa e pressão arterial de repouso em mulheres sedentárias. Revista Brasileira de Cardiologia, Rio de Janeiro, v. 24, n. 3, p. 163-168, 2011.

TORIMOTO-SASAI, Yasuko et al. Female family caregivers face a higher risk of hypertension and lowered estimated glomerular filtration rates: a cross-sectional, comparative study. BMC Public Health, v. 177, n. 15, p. 1-10, 2015

WORLD HEALTH ORGANIZATION (WHO). Physical status: the use and interpretation of anthropometry. Report of a WHO Expert Committee. Geneva: World Health Organization, 1995. (WHO Technical Report Series, 854).

Data de Submissão: 16/05/2018

Data de Aprovação: 29/03/2019 
